\title{
NOTA
}

\section{DISTRIBUIÇÃO E INCERTEZA DA ACIDEZ DE UM LATOSSOLO VERMELHO-AMARELO HÚMICO SOB CULTIVO DE CAFÉ(1)}

\author{
Samuel de Assis Silva ${ }^{(2)}$, Julião Soares de Souza Lima ${ }^{(3)}$, Gustavo \\ Soares de Souza ${ }^{(2)}$ \& Alexandre Candido Xavier ${ }^{(4)}$
}

\begin{abstract}
RESUMO
Com o desenvolvimento da agricultura, a utilização de novas alternativas na avaliação das propriedades que influenciam o rendimento das plantas tornou-se indispensável para melhor manejar o sistema agrícola. O objetivo deste trabalho foi utilizar uma ponderação por meio da modelagem fuzzy para estudar, com base em atributos químicos do solo, a propagação de incertezas da acidez de um Latossolo Vermelho-Amarelo húmico cultivado com café arábica. As amostragens de solo foram realizadas na profundidade de $0-20 \mathrm{~cm}$, em uma malha totalizando 50 pontos. Os atributos avaliados foram: $\mathrm{pH}$ em $\mathrm{H}_{2} \mathrm{O}$, acidez trocável ( $\left.\mathrm{Al}^{3+}\right)$, acidez potencial $(\mathrm{H}+\mathrm{Al})$ e saturação por $\mathrm{Al}(\mathrm{m})$. Os dados foram analisados pela estatística descritiva e pela geoestatística. Utilizou-se um sistema de classificação fuzzy e os atributos descritos para inferir sobre a acidez do solo. A lógica fuzzy, pelo algoritmo utilizado, teve um bom desempenho na caracterização e no mapeamento das incertezas da acidez do solo em questão.
\end{abstract}

Termos de indexação: geoestatística; krigagem; lógica nebulosa; manejo do solo.

\footnotetext{
(1) Recebido para publicação em setembro de 2008 e aprovado em abril de 2009.

${ }^{(2)}$ Mestre em Produção Vegetal, Universidade Federal do Espírito Santo - UFES. Caixa Postal 16, CEP 29500-000 Alegre (ES). E-mail: samuel-assis@hotmail.com

${ }^{(3)}$ Professor Associado, UFES. E-mail: juliaosslima@cca.ufes.br

(4) Professor Adjunto, UFES. E-mail: xavier@cca.ufes.br
} 


\title{
SUMMARY: DISTRIBUTION AND UNCERTAINTY OF ACIDITY OF AN OXISOL UNDER COFFEE CULTIVATION
}

\begin{abstract}
With the development of agriculture, the use of new tools for the evaluation of the properties related to plant yields have become essential to improve the management of agricultural systems. The purpose of this study was to use a fuzzy model to investigate, based on soil chemical properties, the propagation of uncertainty of the acidity of a humic Red-Yellow Latosol (Oxisol) under Arabic coffee plantation. The soil was sampled at a depth of $0-0.2 \mathrm{~m}$, in a grid, totaling 50 sampling points. The following attributes were determined: $\mathrm{pH}$ in $\mathrm{H}_{2} \mathrm{O}$, exchangeable acidity $\left(A l^{3+}\right)$, potential acidity $(H+A l)$ and $A l$ saturation $(m)$. The data were analyzed by descriptive statistics and geostatistics. A fuzzy classification system was used based on the attributes described to infer on the soil acidity. Fuzzy logic, with the algorithm used, was efficient to characterize and map the uncertainties of acidity of the soil under study.
\end{abstract}

Index terms: geostatistics; kriging; hazy logic; soil management.

\section{INTRODUÇÃO}

Os solos podem ser naturalmente ácidos devido à pobreza em bases no material de origem ou a processos de formação que favorecem a remoção de elementos de reação básicos como $\mathrm{K}, \mathrm{Ca}, \mathrm{Mg}, \mathrm{Na}$, etc. A acidificação inicia-se ou acentua-se, devido à remoção de bases do solo e, também, à ação de cultivos e a adubações que, dependendo da forma de condução, podem levar a aumentos significativos dos níveis de acidez (Sousa \& Lobato, 2004).

As culturas e mesmo as variedades ou cultivares variam muito na capacidade de tolerância à acidez $\mathrm{e}$ à disponibilidade de nutrientes. Dessa forma, as classes de fertilidade devem ser interpretadas, considerando as exigências específicas de cada cultivo agrícola (Martins, 2005).

Avanços na agropecuária têm mostrado a importância de novas avaliações das propriedades que alteram o rendimento das culturas, com o intuito de otimizar o aproveitamento de recursos e diminuir custos (Carvalho et al., 2002). Técnicas de análise que objetivem relacionar causas e efeitos e que considerem a natureza dos processos agrícolas, tendo em vista os modelos incertos associados à dinâmica desses processos, devem ser adotadas no gerenciamento da condução das culturas.

Nos últimos anos, observa-se o desenvolvimento de modelos quantitativos de predição espacial, geralmente derivados da geoestatística e de métodos como a lógica fuzzy (McBratney et al., 2000).

A lógica fuzzy objetiva modelar, de modo aproximado, o raciocínio humano, visando manipular informações em um ambiente de incerteza, fornecendo uma resposta aproximada para uma questão baseada em um conhecimento inexato, incompleto ou não totalmente confiável (Meirelles et al., 2007).
Essa técnica tem como apoio a teoria dos conjuntos fuzzy para sua representação e, utilizando expressões linguísticas na sua aplicação, definem-se conjuntos aos quais os valores são alocados com diferentes graus de pertinência entre falso e verdadeiro, ou seja, um elemento pode ou não pertencer a determinado conjunto fuzzy. Caso pertença, estabelece-se o grau de pertinência com que isso acontece (Bönisch et al., 2004).

A modelagem fuzzy tem sido amplamente utilizada por basear-se na caracterização de classes que não possuem, ou não podem definir limites rígidos entre si (Burrough \& Mcdonnell, 1998), como acontece nas classes de fertilidade do solo, sendo indicada para lidar com ambiguidades, abstrações e ambivalências de modelos matemáticos complexos que representam limites difusos comuns em processos naturais (Bönisch et al., 2004).

O objetivo desse trabalho foi utilizar uma ponderação por meio da modelagem fuzzy para estudar, com base em atributos químicos do solo, a propagação de incertezas da acidez de um Latossolo Vermelho-Amarelo húmico cultivado com café arábica.

\section{MATERIAL E MÉTODOS}

O trabalho foi conduzido em uma área da fazenda Jaguaraí no município de Reduto, na região leste do Estado de Minas Gerais, a $20^{\circ} 45^{\prime} 45,4$ "de latitude $\mathrm{S}$ e $41^{\circ} 32^{\prime} 9,75$ " de longitude W. A área, com diferença de nível acentuada e $30^{\circ}$ de declividade, vem sendo cultivada há cinco anos com Coffea arabica L., variedade Catuaí, no espaçamento de $2,0 \times 0,60 \mathrm{~m}$, em um Latossolo Vermelho-Amarelo húmico, com horizonte A bastante espesso e rico em matéria orgânica, conforme Embrapa (1999). 
As amostragens de solo foram realizadas em uma malha irregular totalizando 50 pontos georreferenciados (Figura 1), com as amostras coletadas na projeção da copa de três plantas (constituindo o ponto de amostragem), na profundidade de $0-20 \mathrm{~cm}$.

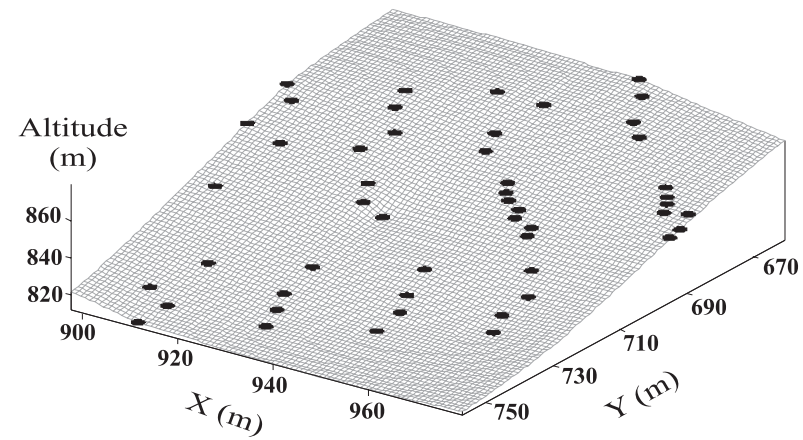

Figura 1. Modelo digital de elevação (MDE) da área em estudo com a distribuição dos pontos amostrais.

Os atributos avaliados foram: $\mathrm{pH}$ em $\mathrm{H}_{2} \mathrm{O}$, acidez trocável $\left(\mathrm{Al}^{3+}\right)$, acidez potencial $(\mathrm{H}+\mathrm{Al})$ e saturação por $\mathrm{Al}(\mathrm{m})$, e os valores encontrados foram analisados por meio das medidas de posição e dispersão da estatística descritiva e exploratória, bem como pelo teste Shapiro-Wilk's $(\mathrm{p}<0,05)$ para testar a normalidade.

A análise da dependência espacial foi feita pela Geoestatística, com auxílio do software $\mathrm{GS}^{+}$ (Robertson, 1998), que realiza os cálculos das semivariâncias amostrais, cuja expressão pode ser encontrada em Vieira et al. (1983):

$$
\gamma^{*}(h)=\frac{1}{2 N(h)} \sum_{i=1}^{N(h)}\left[z\left(x_{i}\right)-z\left(x_{i}+h\right)\right]^{2}
$$

em que $\mathrm{N}(\mathrm{h})$ é o número de pares amostrais $[\mathrm{z}(\mathrm{xi})$; $z(x i+h)]$ separados pelo vetor $h$, sendo $z(x i)$ e $z(x i+h)$ os valores numéricos observados do atributo analisado, para dois pontos $\mathrm{xi}$ e $\mathrm{xi}+\mathrm{h}$ separados pelo vetor $\mathrm{h}$. $\mathrm{O}$ semivariograma é representado pelo gráfico de $\mathrm{g}(\mathrm{h})$ função de h. Após o ajuste de um modelo matemático aos valores calculados de $\mathrm{g}(\mathrm{h})$, são definidos os parâmetros do modelo teórico para o semivariograma (efeito pepita, Co; alcance da dependência espacial, a; e patamar, C). Amostras separadas por distâncias menores que o alcance são espacialmente correlacionadas, ao passo que as separadas por distâncias maiores não o são.

Para análise do índice de dependência espacial (IDE), foram utilizados a relação $\mathrm{C} /\left(\mathrm{C}_{0}+\mathrm{C}\right)$ e os intervalos propostos por Zimback (2001), que consideram as dependências espaciais fraca (IDE $<25 \%$ ); moderada ( $25 \% \leq$ IDE $<75 \%$ ) e forte (IDE $\geq 75 \%$ ).

Na determinação da dependência espacial, utilizouse o exame de semivariogramas, por meio do programa
$\mathrm{GS}^{+}$(Robertson, 1998). Em caso de dúvida entre mais de um modelo para o mesmo semivariograma, utilizou-se o critério dos mínimos quadrados, optandose, na seleção, pelos modelos com maior valor de $\mathrm{R}^{2}$ (coeficiente de determinação), menor SQR (soma de quadrado dos resíduos) e maior valor do coeficiente de correlação obtido pelo método de validação cruzada (Guimarães, 2000).

Visando testar o efeito de funções de álgebra de mapas na propagação de incertezas associadas às representações numéricas, os atributos numéricos $\mathrm{pH}$, $\mathrm{Al}^{3+}, \mathrm{H}+\mathrm{Al}$ e $\mathrm{m}$ foram integrados para espacialização da acidez. Nesta etapa, foi necessário, inicialmente, expressar cada atributo de acordo com as classes de acidez (Quadro 1).

As representações desses atributos foram submetidas a uma classificação contínua utilizando o mapeamento fuzzy. A função da associação escolhida para os valores de $\mathrm{Al}^{3+}, \mathrm{H}+\mathrm{Al}$ e $\mathrm{m}$ foi a linear descrita por Bönisch et al. (2004). Para os valores de $\mathrm{pH}$, utilizou-se a função exponencial apresentada por Meirelles et al. (2007).

- Conjunto de dados de $\mathrm{Al}^{3+}, \mathrm{H}+\mathrm{Al}$ e m

$$
\begin{array}{ll}
\operatorname{MF}_{A}(Z)=0 & \text { se } z<q \\
M_{A}(Z)=(-1 / \alpha) /(z-r) & \text { se } q<z<r \\
M_{A}(Z)=1 & \text { se } z>r
\end{array}
$$

- Conjunto de dados de $\mathrm{pH}$

$$
\begin{array}{ll}
M_{A}(Z)=1 /\left(1+d(z-p)^{2}\right) & \text { se } z<p \\
M_{A}(Z)=1 & \text { se } p<z<q \\
M_{A}(Z)=1 /\left(1+d(z-q)^{2}\right) & \text { se } z>q
\end{array}
$$

em que $\mathrm{MF}_{\mathrm{A}}=$ função pertinência da variável $\mathrm{Z}$; $\alpha=$ r-q; d = q-p e p, q e r são valores dos limites de classe de dois conjuntos fuzzy pertencentes a um conjunto A.

A zona de transição fuzzy é dada pela inclinação das retas para o conjunto A com valores decrescentes $\left(\mathrm{Al}^{3+}, \mathrm{H}+\mathrm{Al}\right.$ e $\left.\mathrm{m}\right)$ e com valores exponenciais $(\mathrm{pH})$.

\section{Quadro 1. Critérios para determinação das classes de acidez $^{(1)}$ do solo}

\begin{tabular}{lccc}
\hline \multirow{2}{*}{ Atributo } & \multicolumn{3}{c}{ Classe de acidez } \\
\cline { 2 - 4 } & Baixo & Médio & Alto \\
\hline $\mathrm{pH}\left(\mathrm{em} \mathrm{H}_{2} \mathrm{O}\right)$ & $<5,50$ & $5,50-6,00$ & $>6,00$ \\
$\mathrm{Al}^{3+}\left(\mathrm{cmol}_{\mathrm{c}} \mathrm{dm}^{-3}\right)$ & $<0,50$ & $0,50-1,00$ & $>1,00$ \\
$\mathrm{H}+\mathrm{Al}\left(\mathrm{cmol}_{\mathrm{c}} \mathrm{dm}^{-3}\right)$ & $<2,50$ & $2,50-5,00$ & $>5,00$ \\
$\mathrm{~m}(\%)$ & $<30,00$ & $30,00-50,00$ & $>50,00$ \\
\hline
\end{tabular}

(1) Classes definidas segundo Alvarez V. et al. (1999). 
Os valores dos parâmetros $\mathrm{p}$, q e r foram definidos em função dos valores dos limites dos intervalos dos atributos, que foram relacionados com a classe de acidez baixa (q e r) e com uma acidez elevada (p) (Quadro 2).

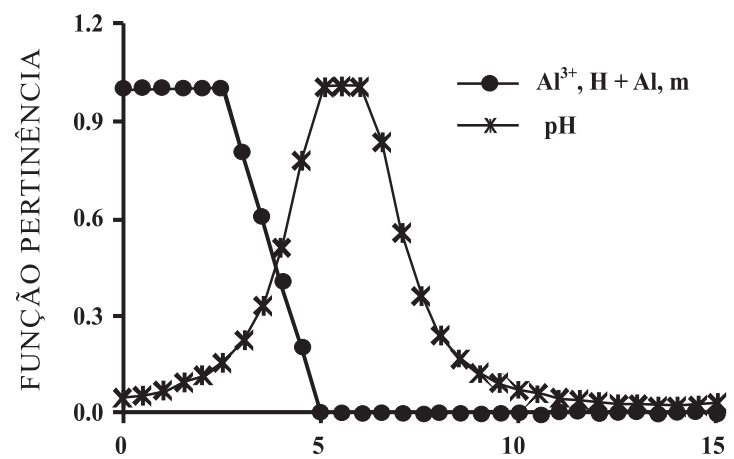

Figura 2. Conjuntos fuzzy para dados com valores com comportamento decrescente e exponencial (adaptado de Burrough \& Mcdonnell, 1998).

Quadro 2. Funções lineares fuzzy utilizadas na classificação contínua das representações dos níveis de: $\mathrm{pH}$ em $\mathrm{H}_{2} \mathrm{O}$, acidez trocável $\left(\mathrm{Al}^{3+}\right)$, acidez potencial $(\mathrm{H}+\mathrm{Al})$ e saturação por alumínio (m)

\section{pH em $\mathrm{H}_{2} \mathrm{O}$}

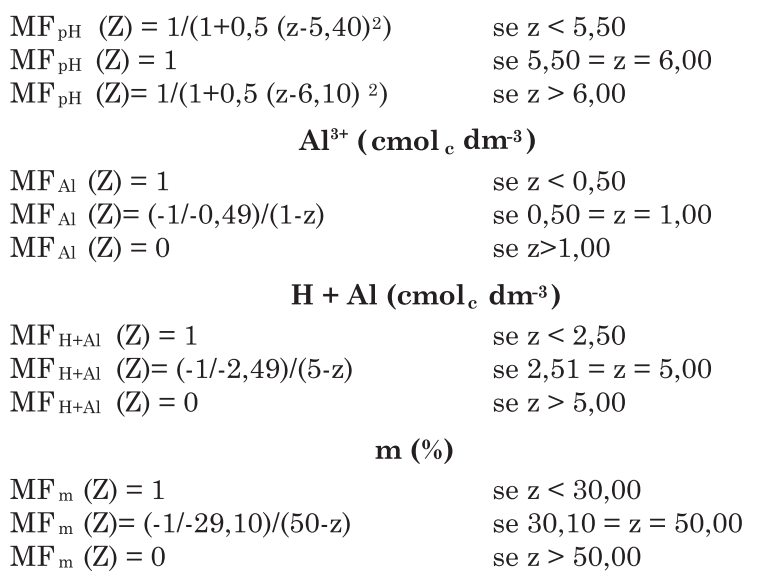

$\mathrm{O}$ efeito combinado dos atributos foi medido utilizando-se funções de álgebra de mapas por meio do operador fuzzy de soma ponderada. Foi atribuído peso igual para todas as representações, ou seja, considerou-se que os atributos contribuíram, de forma equitativa, na estimativa da acidez do solo. Dessa forma, a operação fuzzy se tornou uma média simples das representações.

\section{RESULTADOS E DISCUSSÃO}

Os resultados referentes à análise descritiva para o $\mathrm{pH}$ em $\mathrm{H}_{2} \mathrm{O}, \mathrm{Al}^{3+}\left(\mathrm{cmol}_{\mathrm{c}} \mathrm{dm}^{-3}\right), \mathrm{H}+\mathrm{Al}\left(\mathrm{cmol}_{\mathrm{c}} \mathrm{dm}^{-3}\right) \mathrm{e}$ $\mathrm{m}(\%)$ do solo são apresentados no quadro 3 . Tal análise, apesar de considerar os dados espacialmente independentes, tem a função de analisar seu comportamento geral e identificar influências que prejudicam a análise geoestatística.

Analisando o coeficiente de curtose, com exceção do $\mathrm{H}+\mathrm{Al}$, os atributos apresentaram distribuição platicúrtica, porém com achatamento reduzido da curva de distribuição normal, uma vez que esses valores encontram-se próximos de zero.

Considerando os coeficientes de variação $(\mathrm{CV}) \mathrm{em}$ valores baixos ( $\mathrm{CV}<12 \%)$; médios $(12 \%<\mathrm{CV}$ $<60 \%$ ) e altos (CV > $60 \%$ ), segundo critérios de Warrick \& Nielsen (1980), o pH apresenta baixa variação. Resultados semelhantes foram encontrados por Silva et al. (2003), Souza et al. (2004) e Zanão Júnior et al. (2007). O Al ${ }^{3+}$ e o $\mathrm{H}+\mathrm{Al}$ apresentaram variação mediana, e a saturação por $\mathrm{Al}(\mathrm{m})$, variação elevada, justificado pela sua maior amplitude total, sugerindo influência direta dos extremos na sua variação. Souza (1992) afirma existir correlação diretamente proporcional entre a amplitude dos dados e o coeficiente de variação, o que foi constatado neste estudo.

Pelos valores médios dos atributos químicos do solo e com base na classificação apresentada por Alvarez V. et al. (1999), para o Estado de Minas Gerais, os níveis de $\mathrm{pH}$ e $\mathrm{Al}^{3+}$ são classificados como médios, enquanto os valores de m enquadram-se na classe de

Quadro 3. Estatísticas da distribuição do $\mathrm{pH}$ em $\mathrm{H}_{2} \mathrm{O}$, e do teor de acidez trocável (Al ${ }^{3+}$ ), acidez potencial $(\mathrm{H}+\mathrm{Al})$ e saturação por alumínio (m)

\begin{tabular}{|c|c|c|c|c|c|c|c|c|c|}
\hline Atributo & Média & Mediana & Menor & Maior & CV (\%) & $\mathbf{s}$ & $\mathrm{C}_{\mathrm{s}}$ & $\mathbf{C}_{\mathbf{k}}$ & $\mathbf{w}$ \\
\hline $\mathrm{pH}$ & 5,10 & 5,08 & 4,62 & 5,62 & 4,79 & 0,24 & 0,10 & $-0,48$ & $\mathrm{~ns}$ \\
\hline $\mathrm{Al}^{3+}$ & 0,54 & 0,50 & 0,00 & 1,30 & 58,95 & 0,32 & 0,08 & $-0,51$ & $\mathrm{~ns}$ \\
\hline $\mathrm{H}+\mathrm{Al}$ & 5,38 & 5,40 & 3,65 & 7,43 & 14,84 & 0,80 & 0,23 & 0,05 & $\mathrm{~ns}$ \\
\hline $\mathrm{m}$ & 24,69 & 23,60 & 0,00 & 64,70 & 64,94 & 16,04 & 0,32 & $-0,43$ & $\mathrm{~ns}$ \\
\hline
\end{tabular}

$\mathrm{Al}\left(\mathrm{cmol}_{\mathrm{c}} \mathrm{dm}^{-3}\right) ; \mathrm{H}+\mathrm{Al}\left(\mathrm{cmol}_{\mathrm{c}} \mathrm{dm}^{-3}\right) ; \mathrm{m}(\%) ; \mathrm{CV}$ : coeficiente de variação; s: desvio padrão; $\mathrm{C}_{\mathrm{s}}$ : coeficiente de assimetria; $\mathrm{C}_{\mathrm{k}}$ : coeficiente de curtose; ns: distribuição normal pelo teste Shapiro-Wilk's a $5 \%$. 
baixa saturação. A acidez potencial $(\mathrm{H}+\mathrm{Al})$ encontrase classificada como alta.

Constata-se que todos os atributos apresentaram distribuição normal, analisada pelo teste ShapiroWilk's a $5 \%$, sendo esta distribuição simétrica, uma vez que os coeficientes de assimetria são próximos de zero, implicando valores de média e mediana coincidentes (Quadro 3). Segundo Isaaks \& Srivastava (1989), mais importante que a normalidade dos dados é a ocorrência ou não do chamado efeito proporcional, ou seja, que a média e a variabilidade sejam constantes na área de estudo (Figura 4), em que se observa que os dados estão distribuídos homogeneamente em toda a área, não havendo regiões com concentração de valores altos ou baixos em zonas específicas da área estudada, ocorrendo assim a estacionaridade isotrópica - o que é essencial na aplicação da Geoestatística.

Devido à configuração da área (formato retangular com predomínio de pontos na direção norte-sul), assumiu-se a hipótese de isotropia dos dados para construção do semivariograma e posterior krigagem, uma vez que não é possível visualizar se há ou não anisotropia dos dados.

Analisando os semivariogramas (Figura 4), observa-se que todos os atributos apresentaram dependência espacial. Como os semivariogramas apresentaram patamares bem definidos, assumiu-se, nesse caso, estacionaridade intrínseca, uma vez que não se verificou tendência de variação dos atributos com as direções (Figura 3).

Ajustou-se aos valores de $\mathrm{pH}$ e $\mathrm{H}+\mathrm{Al}$ o modelo esférico. $\mathrm{Ao}^{3+} l^{3+}$, o modelo que melhor se ajustou foi o gaussiano. Os alcances dos semivariogramas

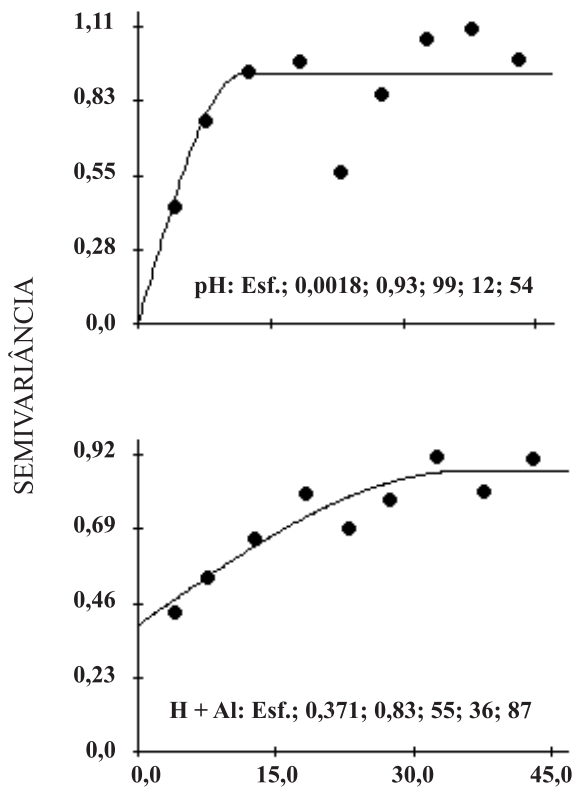

variaram de $12 \mathrm{~m}$ para o $\mathrm{pH}$ e de $56 \mathrm{~m}$ para o $\mathrm{Al}^{3+}$. O valor de alcance tem aplicação direta no plano de amostragem em agricultura de precisão, pois indica
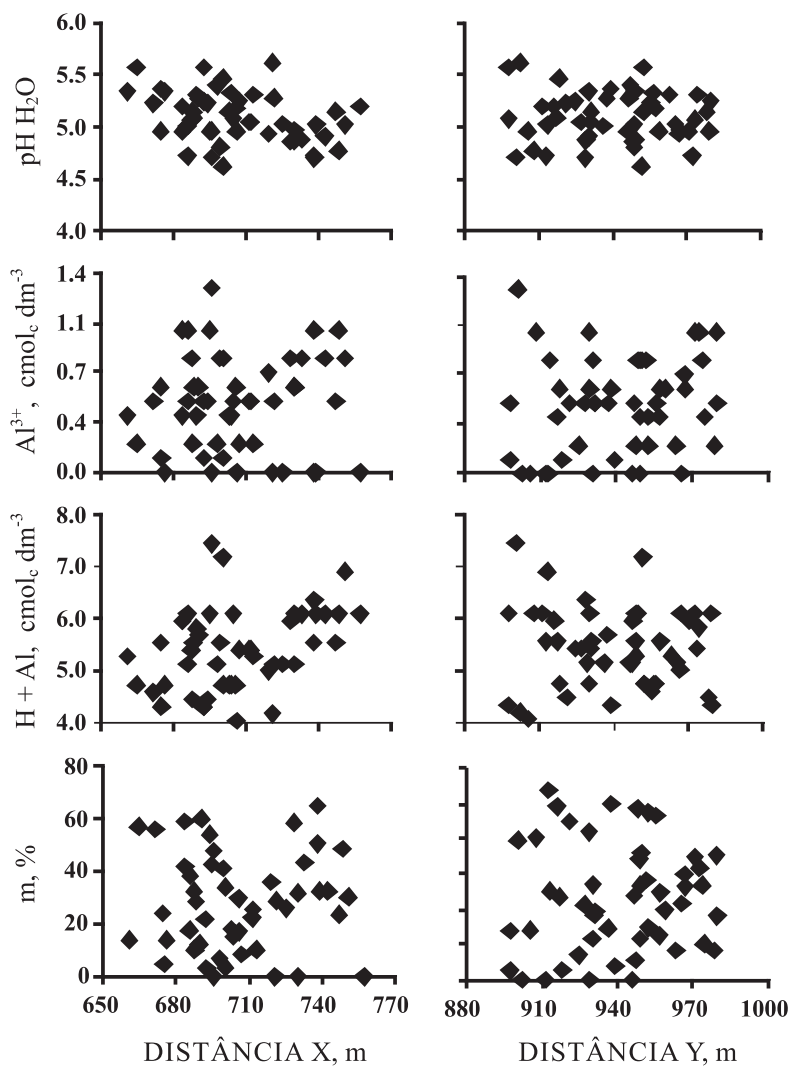

Figura 3. Dispersão dos dados de acidez em função das coordenadas $\mathrm{X}$ e $\mathrm{Y}$.
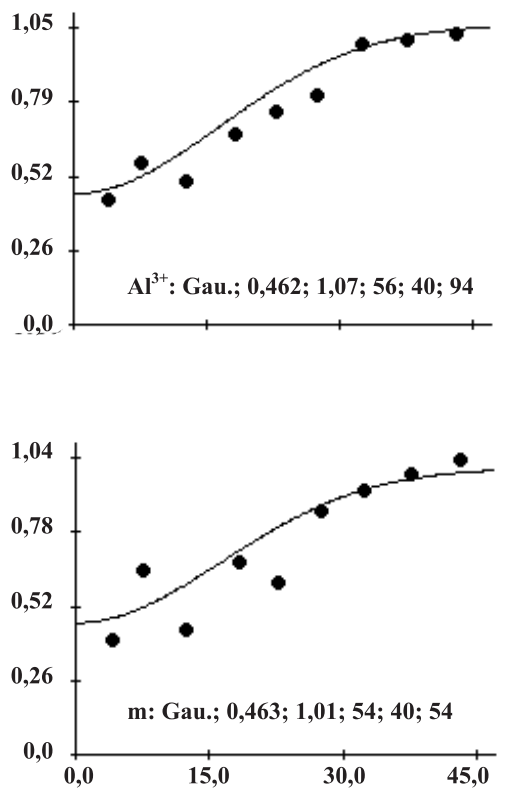

DISTÂNCIA, m

Figura 4. Modelos e parâmetros $\left(\mathrm{C}_{0} ; \mathrm{C}_{0}+\mathrm{C}_{1} ; \mathrm{IDE} ; \mathrm{A}_{0}\right.$ e $\left.\mathrm{R}^{2}\right)$ dos semivariogramas escalonados para os atributos químicos do solo. 
a distância máxima que uma variável está correlacionada espacialmente (Vieira, 2000), ou seja, determinações realizadas a distâncias maiores que o alcance têm distribuição aleatória e, por isso, são independentes entre si.

$\mathrm{O}$ índice de dependência espacial (IDE) mostra moderada dependência para $\mathrm{Al}^{3+}, \mathrm{H}+\mathrm{Al}$ e $\mathrm{m}$, e o $\mathrm{pH}$ apresenta forte dependência espacial, de acordo com classificação proposta por Zimback (2001).

Com exceção do $\mathrm{pH}$, os valores do efeito pepita $\left(\mathrm{C}_{0}\right)$ estão distantes de zero, o que reduz a exatidão de estimativas, por meio da krigagem, de valores em locais não medidos, uma vez que o $\mathrm{C}_{0}$ representa o componente da variabilidade espacial que não pode ser relacionado com uma causa específica (variabilidade ao acaso), ou seja, quanto menor a variação ao acaso, mais exata é a estimativa (Vieira, 2000).

A espacialização das possibilidades (incertezas) dos atributos, onde se observa que a quase totalidade da área apresenta valores de pertinência próximos de 1 para o $\mathrm{pH}$, demonstrando baixa atividade de íons $\mathrm{H}^{+}$ em solução (Figura 5).

Graus de saturação de $\mathrm{Al}^{3+}$ e, ou, deficiência de $\mathrm{Ca}$, frequentes em situações de $\mathrm{pH}$ baixos, restringem o crescimento do sistema radicular das plantas, tanto das camadas superficiais quanto subsuperficiais (Silva $\&$ Souza, 1998).
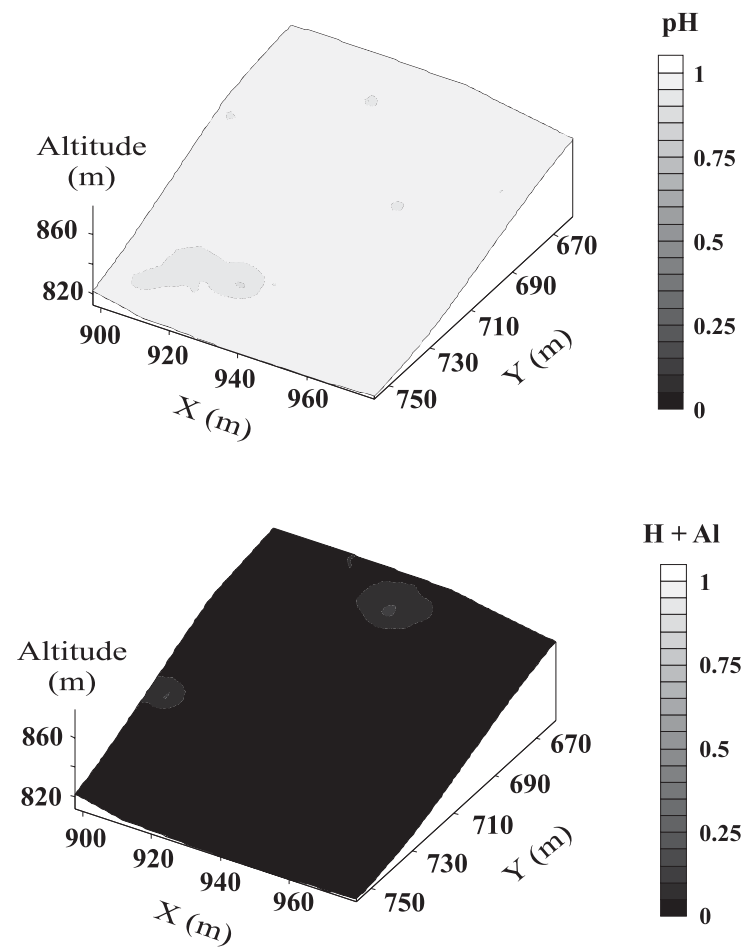

$\mathrm{O} \mathrm{Al}^{3+}$ segue o comportamento dos atributos supra discutidos, com valores de pertinência próximos de 1 , indicando sua precipitação na forma $\mathrm{Al}(\mathrm{OH})_{3}$, causando baixa concentração desse elemento na solução do solo, o que favorece o crescimento do sistema radicular das plantas, uma vez que o efeito primário da sua toxidez manifesta-se, geralmente, bloqueando o crescimento radicular (Epstein \& Bloom, 2006).

A acidez potencial $(\mathrm{H}+\mathrm{Al})$, divergindo dos demais atributos, apresentou valores de pertinência próximos de zero. Como esta acidez é representada por $\mathrm{H} \mathrm{e} \mathrm{Al}$ na fase sólida do solo em equilíbrio com os íons da solução, sendo liberados quando há um aumento do $\mathrm{pH}$, sugere-se redução da saturação por bases e elevado poder tamponante do solo, ou seja, maior resistência em ter o valor de seu $\mathrm{pH}$ alterado (Camargos, 2005). Essa característica é comum em solos ricos em matéria orgânica como os húmicos (Jacques, 2003).

A área em estudo apresenta graus de pertinência elevados para a acidez do solo, demonstrando que, de modo geral, a acidez na área é reduzida (Figura 6).

Os valores de pertinência indicam variação importante na área estudada e demonstram, de forma geral, boas possibilidades de crescimento da variedade Catuaí na área, uma vez que esta variedade é muito sensível à acidez, principalmente ao $\mathrm{Al}$ trocável (Rodrigues et al., 2006).
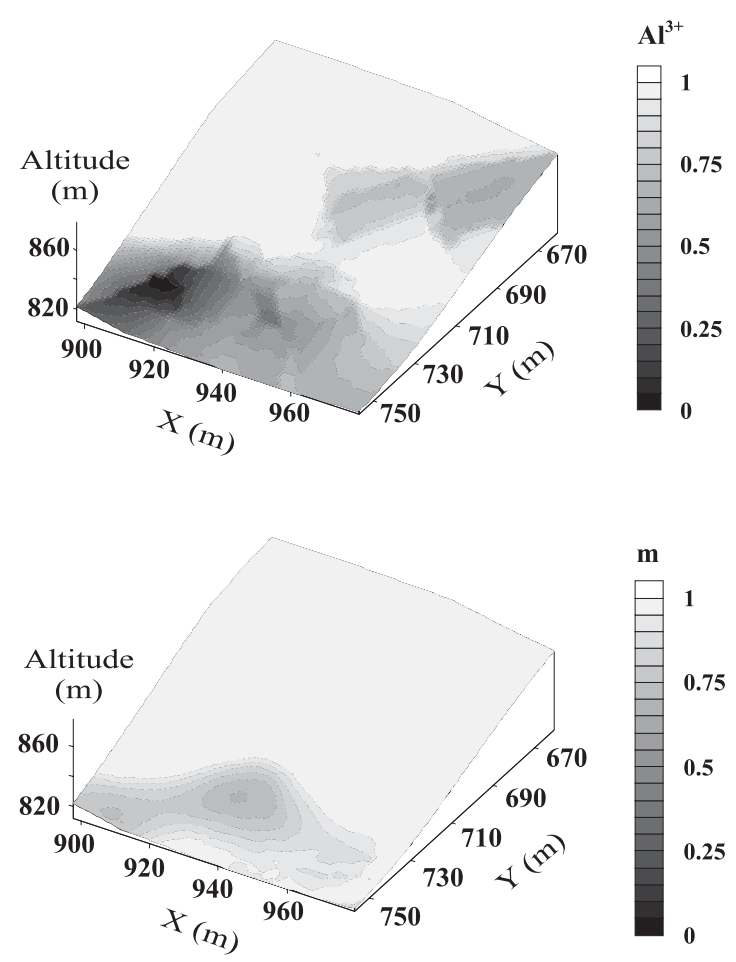

Figura 5. Mapas de classificação fuzzy para os atributos $\mathrm{pH}, \mathrm{Al}^{3+}, \mathrm{H}+\mathrm{Al}$ e m. 


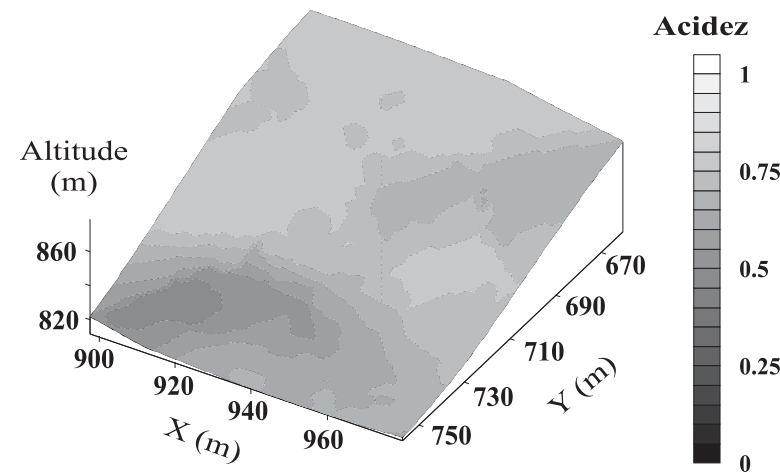

Figura 6. Mapa da ponderação da acidez do solo, com base nos atributos avaliados.

\section{CONCLUSÕES}

1. A lógica fuzzy, pelo algoritmo utilizado, teve um bom desempenho para caracterizar e mapear as incertezas da acidez do solo em questão.

2. A área em estudo apresentou graus de pertinência elevados para a acidez do solo, indicando acidez reduzida na área.

\section{AGRADECIMENTOS}

Á CAPES, pelo auxílio por meio da concessão de bolsa de mestrado.

\section{LITERATURA CITADA}

ALVAREZ V., V.H.; NOVAIS, R.F.; BARROS, N.F.; CANTARUTTI, R.B. \& LOPES, A.S. Interpretação dos resultados das análises de solos. In: RIBEIRO, A.C.; GUIMARÃES, P.T.G. \& ALVAREZ V., V.H., eds. Recomendações para uso de corretivos e fertilizantes em Minas Gerais. $5^{\text {a }}$ Aproximação. Viçosa, MG, Comissão de Fertilidade do Solo do Estado de Minas Gerais - CFSEMG, 1999. p.25-32.

BÖNISCH, S.; LOPES ASSAD, M.L.; CÂMARA, G. \& MONTEIRO, A.M.V. Representação e propagação de incertezas em dados de solos, 1- Atributos categóricos. R. Bras. Ci. Solo, 28:21-32, 2004.

BURROUGH, P.A. \& McDONNELL, R.A. Principles of geographic information systems. New York, Oxford University Press, 1998. 333p.

CAMARGOS, S.L. Acidez do solo e calagem (reação do solo). Material didático. Cuiabá, Universidade Federal do Mato Grosso, Faculdade de Agronomia e Medicina Veterinária, 2005. 26p.
CARVALHO, J.R.P.; SILVEIRA, P.M. \& VIEIRA, S.R. Geoestatística na determinação da variabilidade espacial de características químicas do solo sob diferentes preparos. Pesq. Agropec. Bras., 37:1151-1159, 2002.

EMPRESA BRASILEIRA DE PESQUISA AGROPECUÁRIA EMBRAPA. Centro Nacional de Pesquisa de Solos, Sistema brasileiro de classificação de solos. Rio de Janeiro, 1999. 412p.

EPSTEIN, E. \& BLOOM, A.J. Nutrição mineral de plantas: Princípios e perspectivas. 2.ed. Londrina, Planta, 2006. 403p.

GUIMARÃES, E.C. Variabilidade espacial de atributos de uma Latossolo Vermelho-Escuro textura argilosa da região do cerrado, submetido ao plantio direto e ao plantio convencional. Campinas, Universidade Estadual de Campinas, 2000. 85p. (Tese de Doutorado)

ISAAKS, E.H. \& SRIVASTAVA, R.M. An introdution to applied geostatistics. New York, Oxford University Press, 1989. $561 \mathrm{p}$.

JACQUES, A.V.A. A queima das pastagens naturais - efeitos sobre o solo e a vegetação. Ci. Rural, 33:145-152, 2003.

MARTINS, C.E. Práticas agrícolas relacionadas à calagem do solo. Juiz de Fora, Embrapa - MG, 2005. 6p. (Comunicado Técnico)

McBRATNEY, A.B.; ODEH, I.O.A.; BISHOP, T.F.A.; DUNBAR, M.S. \& SHATAR, T.M. An overview of pedometric techniques for use in soil survey. Geoderma, 97:293-327, 2000 .

MEIRELLES, M.S.P.; MOREIRA, F.R. \& CAMARA, G. Técnicas de inferência espacial. In: MEIRELLES, M.S.P.; CAMARA, G. \& ALMEIDA, C.M., eds. Geomática: Modelos e aplicações ambientais. Brasilia, Embrapa, Informação Tecnológica, 2007. 593p.

ROBERTSON, G.P. GS ${ }^{+}$: Geostatistics for the environmental sciences - $\mathrm{GS}^{+}$User's guide. Plainwell, Gamma Desing Software, 1998. 152p.

RODRIGUES, L.A.; MARTINEZ, H.E.P.; NEVES, J.C.L.; NOVAIS, R.F. \& MENDONÇA, S.M. Respostas nutricionais de cafeeiros catuaí e icatu a doses de calcário em subsuperfície. R. Bras. Ci. Solo, 30:985-995, 2006.

SILVA, C.R. \& SOUZA, Z.M. Eficiência do uso de nutrientes em solos ácidos: Manejo de nutrientes e uso pelas plantas. Ilha Solteira, Universidade Estadual de São Paulo, 1998. (Caderno Didático)

SILVA, V.R.; REICHERT, J.M.; STORCK, L. \& FEIJÓ, S. Variabilidade espacial das características químicas do solo e produtividade de milho em um Argissolo VermelhoAmarelo distrófico arênico. R. Bras. Ci. Solo, 27:10131020, 2003.

SOUSA, D.M. \& LOBATO, E. Cerrado: Correção do solo e adubação. 2.ed. Brasília, Embrapa Informação Tecnológica, 2004. 416p.

SOUZA, L.S. Variabilidade espacial do solo em sistemas de manejo. Porto Alegre, Universidade Federal do Rio Grande do Sul, 1992. 162p. (Tese de Doutorado) 
SOUZA, Z.M.; MARQUES JÚNIOR, J.; PEREIRA, G.T. \& MOREIRA, L.F. Variabilidade espacial do $\mathrm{pH}, \mathrm{Ca}, \mathrm{Mg}$ e V\% do solo em diferentes formas do relevo sob cultivo de cana-de-açúcar. Ci. Rural, 34:32-39, 2004.

VIEIRA, S.R. Geoestatística em estudos de variabilidade espacial do solo. In: NOVAIS, R.F.; ALVAREZ V., V.H. \& SCHAEFER, C.E.G.R., eds. Tópicos em ciência do solo. Viçosa, MG, Sociedade Brasileira de Ciência do Solo, 2000. v.1. p.1-53.

VIEIRA, S.R.; HATFIEL, J.L.; NIELSEN, D.R. \& BIGGAR, J.W. Geoestatistical theory and application to variability of some agronomical properties. Hilgardia, 51:1-75, 1983.
WARRICK, A.W. \& NIELSEN, D.R. Spatial variability of soil physical properties in the field. In: HILLEL, D., ed. Applications of soil physics. New York, Academic Press, 1980. p.319-44.

ZANÃO JÚNIOR, L.A.; LANA, R.M.Q. \& GUIMARÃES, E.C. Variabilidade espacial do $\mathrm{pH}$, teores de matéria orgânica e micronutrientes em profundidades de amostragem num Latossolo Vermelho sob semeadura direta. Ci. Rural, 37:PAGINAS, 2007.

ZIMBACK, C.R.L. Análise espacial de atributos químicos de solos para fins de mapeamento da fertilidade do solo. Botucatu, Universidade Estadual Paulista, 2001. 114p. (Tese de Livre-Docência) 